\title{
Prescribing Practices in 24-hour Supervised Community Residences
}

\section{Introduction}

As part of the inspection of mental health services in 2011, the Inspectorate carried out inspections in nineteen 24 hour supervised community residences. Following on from the review of prescribing practices in approved centres in 2010, the Inspectorate was interested in looking at the prescribing practices in community residences.

\section{Method}

The residences inspected had nursing staff on site 24 hours per day. Inspections were both announced (with 24hrs notice) and unannounced. During the inspections of the residences, copies of prescription kardexes or booklets were collected and information on medications prescribed was documented in the individual report on each residence. The number and percentage of a variety of categories of prescribing practices were described. All information was annonomysied. Other issues including legibility, dates of prescriptions and the use of Medical Council Numbers (MCN) were reviewed.

\section{Description}

Information on medication prescribed was obtained from 18 supervised residences. In all, prescriptions for 208 individual residents were reviewed, with a total of 814 prescriptions of individual medications. A variety of categories of prescribing practices are described, for example, the numbers on regular benzodiazepines (i.e.at least once daily), hypnotics (both benzodiazepine and non benzodiazepine), antipsychotic medication, high dose antipsychotic medication and lithium, to name just a few categories. The results are detailed in the table below.

\section{Findings}

Each medication kardex/booklet was reviewed. Prescriptions were written by the consultant psychiatrist, non consultant hospital doctor (NCHD) or by the general practitioner (GP). The quality of handwriting varied, with most prescriptions being legible; a small number of prescriptions were poorly legible.

Medical Council Numbers (MCN) were not used by the prescribing doctors in the majority of cases.

The generic form of medication was prescribed in most cases, but a significant number of prescriptions (261) were out of date.

Self-medication programmes were in operation in seven residences; no resident was on a self-medication programme in four residences and there was no information regarding this in the reports on the remaining seven residences. 


\section{Medications Prescribed}

Benzodiazepines were prescribed on a regular basis for $25 \%$ of residents, but only two residents (1\%) were prescribed more than one benzodiazepine. Twenty two percent of residents were prescribed a hypnotic and $15 \%$ were prescribed a hypnotic PRN, i.e., if required.

Almost all residents (93\%) were prescribed an antipsychotic medication, either orally or by depot injection, and almost half of all residents were on more than one antipsychotic. One third of residents were on high dose antipsychotic medication and a quarter were prescribed PRN antipsychotics. Forty two percent of residents were on antidepressant medication but only a tiny minority were prescribed more than one antidepressant. There was a low number (11\%) on Lithium. 


\begin{tabular}{|c|c|c|}
\hline NUMBER OF INDIVIDUAL PRESCRIPTIONS: & 208 & $\%$ \\
\hline Number on regular benzodiazepines & 52 & $25 \%$ \\
\hline Number on more than one benzodiazepine & 2 & $1 \%$ \\
\hline Number on PRN benzodiazepines & 63 & $30 \%$ \\
\hline Number on benzodiazepine hypnotics & 17 & $8 \%$ \\
\hline Number on Non benzodiazepine hypnotics & 32 & $15 \%$ \\
\hline Number on PRN hypnotics & 31 & $15 \%$ \\
\hline Number on antipsychotic medication & 193 & $93 \%$ \\
\hline Number on high dose antipsychotic medication & 72 & $34 \%$ \\
\hline Number on more than one antipsychotic medication & 100 & $48 \%$ \\
\hline Number on PRN antipsychotic medication & 54 & $26 \%$ \\
\hline Number on Depot medication & 52 & $25 \%$ \\
\hline Number on antidepressant medication & 87 & $42 \%$ \\
\hline Number on more than one antidepressant & 7 & $3 \%$ \\
\hline Number on antiepileptic medication & 69 & $33 \%$ \\
\hline Number on Lithium & 22 & $11 \%$ \\
\hline
\end{tabular}




\section{Discussion}

To the Inspectorate's knowledge, this was the first review of prescribing practices in supervised residences in mental health services and although the sample was small, it points to some interesting issues.

Prescriptions were for the most part, well written and legible. However, almost one third $(32 \%)$ of prescriptions were out of date. These prescriptions had been written more than six months previously, in some cases in 2007 and in one case the prescription dated back to 2005 . The duration of a prescription is six months. ${ }^{1}$

Although Medical Council guidelines ${ }^{2}$ state that prescriptions written by medical practitioners must include the Medical Council registration number, this was ignored in the vast majority of cases. Self-medication programmes were in use in seven of the residences inspected, and information was lacking in seven others. It was good to note that as part of the rehabilitation processes in community residences such a programme was active, and it is to be hoped that many other services will encourage this practice.

\section{Medication}

Twenty five per cent of residents were prescribed benzodiazepines on a regular basis. This figure is higher than the rate of benzodiazepine use in the general population in Ireland of $11.6 \%{ }^{3}$ in 2000 but much lower than a psychiatric inpatient population in Ireland in 2008 of $64 \%{ }^{4}$. In 1989 , Dunbar et al ${ }^{5}$ found a rate of benzodiazepine prescribing of $10.8 \%$ in the general population, and in 1999 an Irish study by Kirby et al $^{6}$ found a rate of $17 \%$ of elderly living in the community using benzodiazepines.

The rate of use of hypnotics (both benzodiazepine and non-benzodiazepine) in this study was $23 \%$.

Almost all residents were prescribed an antipsychotic medication which may not be surprising as this population of residents was one where residents had enduring metal illness. However, almost $50 \%$ of these residents were also prescribed a second antipsychotic, and in a few cases, a third. The NICE Guidelines ${ }^{7}$ state that: " $A$ patient should not be prescribed more than one antipsychotic drug concurrently, except for short periods when changing from one drug to another"; the exception to this guideline is where a patient is prescribed Clozapine for treatment resistant schizophrenia. In view

\footnotetext{
${ }^{1}$ S.I. No.256/1996 - Medicinal Products (Prescription and Control of Supply) Regulations, 1996:7(2)(6)

2 Guide For Registered Medical Practitioners, Medical Council, 2009

3 "Benzodiazepines: Good Practice Guidelines", DOHC, 2002

${ }^{4}$ Finnerty, S: "An audit of benzodiazepine and hypnotic prescribing in Irish inpatient psychiatric units", Irish Psychiatrist, Vol 10,4:238-240

${ }^{5}$ Dunbar, G., Perera, M., Jenner, F. "Patterns of benzodiazepine use in Great Britain as measured by a general population survey”. British Journal of Psychiatry 2009;195: s37-s42

${ }^{6}$ Kirby, M., Denihan, A.,Bruce,I.,Radic,A.,Coakley, D., Lalor, B. "Benzodiazepine use in the elderly in the community". International Journal of Geriatric Psychiatry 1999;14:280-284i

${ }^{7}$ National Institute for Health and Clinical Excellence Guidelines,2009
} 
of these recommendations, the use of antipsychotic polypharmacy in the community residences must be regarded as high.

One of the most striking findings of this survey was the number of residents on high dose antipsychotic medication $-34 \%$. A high dose of antipsychotic medication is defined as a total daily dose of antipsychotic medication (either in one or more medications) greater than $100 \%$ of the maximum recommended daily dose. ${ }^{8}$ The Consensus Group of the Royal College of Psychiatrists ${ }^{9}$ on high-dose antipsychotic medication quotes recent prevalence studies indicating a prevalence of $25 \%$ in an acute in-patient population, while a study by Wilkie et al ${ }^{10}$ detected a prevalence rate of $20 \%$, again in an in-patient population. One of the findings of that particular study was that if a patient was on an antipsychotic for more than five years, it was more likely to be prescribed at a high dose. As the population of residents in our survey was a community-based one, one would expect that they were a less unwell group than an inpatient group and therefore should be less likely to require high dose antipsychotics.

Twenty five per cent of residents were prescribed a long acting antipsychotic medication (a depot). This rate is not dissimilar to rates quoted in an article in $2009^{11}$, which cites rates of 30\% (in 1996) and 29\% (in 2003) in out-patient populations in the UK.

\section{Conclusion}

Almost one third of prescriptions were out of date, which suggests that prescriptions were not reviewed regularly by the treating team. Our survey of medications prescribed in supervised community residences showed a higher rate of benzodiazepine prescribing than in elderly populations living in the community or in the general population as a whole, but a much lower rate than an in-patient population in psychiatric units. There was a very low rate of prescribing more than one benzodiazepine. The prescribing of antipsychotic medication was almost universal, with almost half of these residents being prescribed more than one antipsychotic medication. There was a significantly higher rate of high-dose antipsychotic prescribing than was found in two inpatient surveys, and was contrary to the recommendations of the Consensus group of the Royal College of Psychiatrists on high-dose antipsychotic medication. It is suggested that a full review of prescribing practices in community residences should take place.

\footnotetext{
${ }^{8}$ Royal College of Psychiatrists, 2006

${ }^{9}$ Consensus statement on high-dose antipsychotic medication, CR138, Royal College of Psychiatrists.

${ }^{10}$ Wilke, A., Preston, N., Wesby.R. "High dose neuroleptics - who gives them and why?" The Psychiatrist, 2001;25:179-183

${ }^{11}$ Barnes, T., Shingelton-Smith, A., Paton, C. "Antipsychotic long-acting injections: prescribing practice in the UK”. British Journal of Psychiatry, 2009; 195:s37-s42
} 


\section{Recommendations}

1. All prescriptions should be in date.

2. Doctors should use MCNs when writing prescriptions.

3. The use of combined antipsychotics should be reviewed.

4. The use of high-dose antipsychotic medication should be reviewed urgently. 